\title{
A Review on Accuracy of Doppler Ultrasound in Various Knee Joint Pathologies
}

\author{
Nazeeha Waseem $^{1 *}$, Nosheen Arshad ${ }^{2}$, Kainat Saleem ${ }^{3}$, Abid Ali ${ }^{4}$, Rizwan Farooq ${ }^{5}$, Romaisa Sultan ${ }^{6}$ \\ ${ }^{1}$ Medical Imaging Doctor, Department of Radiological Sciences and Medical Imaging, The University of Lahore, Gujrat, University of \\ Lahore, Adjacent Chenab Bridge, Gujrat, Punjab 50700, Pakistan \\ ${ }^{2}$ Lecturer, Department of Radiological Sciences and Medical Imaging, The University of Lahore, Gujrat, University of Lahore, \\ Adjacent Chenab Bridge, Gujrat, Punjab 50700, Pakistan \\ ${ }^{3}$ Medical Imaging Technologist, Islamabad Diagnostic Centre, Cantonment Plaza Bhairh Pully Sialkot Cantt, Sialkot, Punjab, Pakistan \\ ${ }^{4}$ Associate Professor, Department of Allied health Sciences, The University of Lahore, Gujrat, University of Lahore, Adjacent Chenab \\ Bridge, Gujrat, Punjab 50700, Pakistan \\ ${ }^{5}$ Medical Officer, Aziz Bhatti Shaheed Teaching Hospital, Bhimber Rd, Mamada, Mahmada, Gujrat, Punjab, Pakistan \\ ${ }^{6}$ Medical Imaging Technologist, FMH College of Medicine and Dentistry, Shadman Rd, Ichhra Lahore, Punjab 54000, Pakistan
}

DOI: $10.36348 /$ sjmps.2021.v07i02.006

| Received: 29.01.2021 | Accepted: 15.02.2021 | Published: 17.02.2021

*Corresponding author: Nazeeha Waseem

\section{Abstract}

Pain in the knee joint has become one of the most common pathologies that are affecting humans of all age groups in the modern world. Many of the arthropathies are resolved with supportive care and complete tests but some of the diseases require early diagnosis and prompt treatment to prevent any long time effects. Doppler ultrasonography has revolutionized our way of approaching these diseases. It has several advantages over other imaging techniques. It is noninvasive and there is no use of a contrast dye which permits us to use this technique even in those patients who are hyper allergic. There is no use of radiations in ultrasonography. It can detect various minor abnormalities like cartilage thinning, partial or complete tears, synovial joints, abnormalities in the bony structure, inflammation debris in the joint, hematoma, and any ligamentous injury. It also helps us in the localization of the injury which further assist us in several aspects, one of which being the administration of the drugs with the help of an injection at the most optimal site.

Keywords: Doppler Ultrasound, knee joint pathologies, arthropathy, tendonitis.

Copyright (C) 2021 The Author(s): This is an open-access article distributed under the terms of the Creative Commons Attribution 4.0 International License (CC BY-NC 4.0) which permits unrestricted use, distribution, and reproduction in any medium for non-commercial use provided the original author and source are credited.

\section{INTRODUCTION}

Knee joint disorders are one of the most common complaints in the field of orthology affecting people of all age groups [1]. Whether the cause of knee pain is the consequence of aging, excessive pressure, or any other pathological disorder, the knee joint is one of the most vulnerable joints in the human body since it is one of the most diligent joints present throughout the body $[2,3]$. A joint is made up of ligaments, cartilage, bone, and synovial fluid. There are two groups of muscles that coordinate movement at the knee joint. The first one is the quadriceps and second is the hamstring muscles. Some sort of destruction in any of the structures supporting the knee joint can cause knee pain which causes difficulty in walking. Most of the knee problems reported in the elderly are simply because of the normal aging process and continuous wearing and tearing thus poses a great challenge for the clinicians [3-5]. Out of the many diseases, osteoarthritis is the most prevalent disorder in the modern world [6]. The number of patients suffering from osteoarthritis is increasing day by day because of the obesity epidemic and the aging process [7].

Diagnosing the etiology of the knee pain includes a proper medical examination and the clinician may order some imaging tests [8]. Over the course of years, many diagnostic imaging techniques have been improved to assess the exact severity of the disease affecting the fragile structures of the knee joint like magnetic resonance imaging [9, 10], Doppler ultrasonography [11], X-Rays [12], and CT scan. We have come a long way to get as much information as we can by using these techniques and which undoubtedly have helped us a lot in the assessment of the disease as compared to the situation a decade ago but there is still a long way to go. Out of the various imaging techniques used by rheumatologists, the Doppler ultrasonography is the most common imaging technique being used 
these days [13]. Thanks to the advancements being made every day to perfect this technique. By using ultrasonography, we can detect very minute changes in the physiology of the joints which are governing the pathological changes affecting the tendons, ligaments, bones that make the joint, fluid accumulation and inflammation of the joint such as in inflammatory arthropathy, and destruction of the articular surfaces such as those observed in the degenerative type of diseases [14].

The utilization of Doppler ultrasonography has several advantages over other imaging techniques. One of the most beneficial things about this technique is that there is no injection of the contrast dye involved to which some people are allergic to. This is a noninvasive technique. It is readily accessible and is of very low cost as compared to Magnetic resonance imaging. We can also study the structure of the tendons, ligaments, and bones on a micro level. Another huge advantage of using Doppler ultrasonography is that there are no radiations involved in this technique so the chances of malignancies as a result of ultrasonography are almost zero. The chance of the associated complications because of this imaging technique is almost next to nothing. There are some structures in which MRI has the upper hand like menisci, cruciate ligaments, and bone marrow but still ultrasonography can be employed sub-optimally. In this article, we will throw light on how Doppler ultrasonography provides aid in diagnosing various knee disorders and in some cases even treating them $[15,16]$.

\section{Ultrasonography in Various Knee Disorders}

Knee ultrasound is useful if we want to diagnose multiple conditions like cellulitis, abscess of the soft tissue, cellulitis, cystic lesions, joint effusions, and help in differentiating whether the dilation in the knee is because of the cyst or aneurysm, injuries of the muscle like the rupture of the quadriceps tendon, inflammatory conditions affecting the bursa of the knee like prepatellar bursitis, infrapatellar bursitis, etc.

\section{Bursae}

Numerous bursae are present in the knee which serves the function of reducing the friction between the joints. These are various sacs filled with fluid and any condition that compromises the structural integrity of the sac can result in the effusion of the fluid. The joint effusion on an ultrasound can appear as anechoic if the injury is acute or hypoechoic if the injury is containing some debris material. There are four bursae present around the knee joint and all are prone to inflammation.

\section{Prepatellar Bursitis}

Prepatellar bursitis is the bursa present between the skin and the knee and is the most commonly inflamed bursitis of the knee because of its relatively superficial position [17]. Prepatellar bursitis is commonly seen in those people who spend much of their time kneeling, hence the name housemaid's knee. Other risk factors include infectious diseases, rheumatoid arthritis, direct injury to the bursa, or gout [18-20]. Ultrasound helps differentiate inflammation and tendinopathy and also in obtaining needle aspirates from the optimal position [21]. The inflamed bursa appears as an area of hypoechogenicity showing synovial fluid accumulation which is sometimes accompanied by thickened synovium [22].

\section{Knee Tendon Bursitis}

Knee tendon bursitis also known as PES Anserine Bursitis is the inflammation of the bursa that is present between the tibia and the three muscles out of which two are the knee adductors while one is a part of the hamstring muscles namely sartorius, gracilis, and semitendinosus. This type of bursitis is commonly seen in athletes due to overexertion of the hamstring muscles and the usage of inappropriate training techniques [24]. Sonographically, a normal bursa appears to show hyperechogenicity peripherally and hypoechogenicity in the center. In knee tendon bursitis, ultrasound shows a cystic mass and sometimes reveals inflammatory exudates in the bursae [25]. Doppler ultrasonography also plays an effective role in locating the accurate site of the PES anserine bursa for the proper administration of peripheral nerve blockers needed in this disease [26].

\section{Popliteal Cysts}

Popliteal cyst, also called baker's cyst, is a condition in which fluid accumulates in the popliteal fossa. It is the enlargement of the gastrocnemius and semi membranosus bursa resulting in a rigid knee joint which causes pain when there is flexion or full extension of the knee [27]. It is commonly seen in diseases that destroy the articular cartilage or those that result in meniscal tears. The diagnosis of the popliteal cyst is confirmed by using ultrasound or magnetic resonance imaging. It is important to pinpoint the location of the flow of synovial fluid that is being sent from the knee joint into the cyst [28]. There is also a chance of rupture of the cyst which can produce further complications like hemorrhages, loose bodies, or bruising of the leg by leakage of the fluid [29]. The ultrasound detects Baker cyst as a structure filled with fluid on the medial aspect of the popularity of fossa which sometimes crosses the midline when it is large enough. In case of fracture, the inferior aspect of the bursa may show a pointed appearance [30].

\section{Tendinopathies}

One of the major strengths of ultrasonography is assessing the physiological condition of different tendons. We can easily differentiate whether the pathology is being caused by a rupture of the tendon or some inflammation. Identification of the site of tendon rupture by ultrasonography has given some new 
insights and has been proven successful in surgical techniques.

\section{Osgood-Schlatter Disease}

Osgood-Schlatter disease is an inflammatory condition in which there is the formation of a bump over the knee which causes pain when there is exertion over the knee joint [31]. The pathophysiology of the disease includes repeated tension over the growth plate of the upper tibia which causes intermittent pain in the knee joint, especially after exertion. The disease is common in those people in whom there is an overuse of the knee joint like athletes, workers, etc. The goal of ultrasound is to diagnose the disease in its early stages [32]. This disease has some unique features and ultrasonography can show these abnormalities perfectly [33]. Increased swelling over the surface of the tibia, increased vascularity of formation of a new tuberosity on the anterior surface of the tibia will be noticed and is shown by acoustic shadowing on the ultrasound [34, 35].

\section{Jumper's Knee}

This disease is also called patellar tendonitis and it is characterized by symptomatic pain on the anterior surface of the knee. The overuse of the patellar tendon is the pathophysiology of this disease which can lead to tearing of the tendon [36]. The patellar tendon connects the tibia to the patella and straightens out the knee. This disease is most commonly seen in those people who play sports that involve jumping like volleyball and basketball [37]. It is a degenerative disorder rather than an inflammatory disease. Usually, the diagnosis is based upon care for medical history but ultrasound and MRI are sometimes used to confirm the diagnosis [38, 39]. Sonographically, there is the presence of hypoechoic focal lesions located in the proximal insertion of the patellar tendon which represents zones of intratendinous lesions and, also by measuring the flow on power Doppler ultrasonography, we can easily find information about the site of pathology [40, 41].

\section{Rupture of the Quadriceps Tendon}

On a normal ultrasound, parallel rays running across the length of the muscle can be seen which represents the convergence of the four muscles [42]. The quadriceps tendon has three layers. The superficial layer is formed by the rectus femoris muscle. The intermediate layer is formed by vastus medialis and vastus lateralis while the deepest layer is formed by vastus intermedius. The tears in the quadriceps tendon show duality in their nature i.e. partial or complete. Complete tears are the ones in which there is the complete destruction of the soft tissue resulting in the rupture of all three layers of the quadriceps tendon. In contrast to the complete tear, the partial tear represents the rupture of a single or multiple layers. The function of the tendon is the extension of the knee which is lost after the rupture rendering the knee incompetent for straightening ultimately resulting in the loss of a normal gait [43, 44]. There is a tearing or popping sensation accompanied by pain and swelling. By using ultrasonography, not only we can determine the site of the tear but also we can find information about whether the tear is complete or incomplete. On a normal ultrasound, parallel rays running across the length of the muscle can be seen which represents the convergence of the four muscles. Ultrasonogram shows an anechoic area superior to the patella with disrupted quadriceps and $\mathrm{b}$ which it is accompanied by hematoma [45].

\section{Other Tendinopathies}

Other than the aforementioned diseases, some other tendinopathies can also be visualized by using ultrasonography like distal biceps femoris and pes anserinus tendinitis, which are seen mostly in athletic people. Ultrasonography shows thickening of the bands or synovial fluid effusion in the neighborhood of the knee bursae [46].

\section{Ligaments}

There are four ligaments present in the knee joint namely anterior cruciate ligament, posterior cruciate ligament, medial collateral ligament, and lateral collateral ligament. The anterior and posterior cruciate ligament keeps in check the anterior and posterior movement of the knee cruciate ligament helps in stabilizing the knee. Both the cruciates are intracapsular ligaments and appear hypoechoic while the collateral ligaments are outside the joint capsule and appear hyperechoic on an ultrasonogram [47]. Commonly, injuries to the ligaments occur when the knee is stretched or when there is a direct impact on the knee when the foot is in a planted position. Injury to the ligament can be a complete or a partial tear. In a complete tear, there is the presence of hypoechoic granulation tissue whereas, in partial tear, this hypoechogenicity is localized to a small portion.

\section{Anterior Cruciate Ligament Injury}

The anterior cruciate ligament connects the knee to the shin bone and originates from the medial wall of the lateral femoral condyle and inserts into the intercondylar area. Injury to the ACL occurs when the feet stay planted and the knee is stretched in the opposite direction. This results in overstretching of the anterior cruciate ligament. Sonographically, the anterior cruciate ligament appears as a small oval-shaped hypoechoic structure but when there is a tear present in the ligament, there is hypoechoic collection along the intercondylar notch representing a presence of a hematoma [48-50].

\section{Posterior Cruciate Ligament Injury}

The posterior cruciate ligament joins the medial condyle of the femur to the posterior part of the intercondylar area. The PCL is an opposite analog of 
the ACL and prevents the sliding of the femur over the tibia in such a way that tibia is not displaced posteriorly. Falling when the knee is bent while the foot is pointing downwards is a common cause of injury to the ligament and occurs mostly in contact sports [51]. Ultrasound shows this injury as enlargement of the entire posterior ligament and also there is the loss of sharply defined posterior border [52].

\section{Medial Collateral Ligament Injury}

There are two portions of the medial collateral ligament namely the deep and superficial. The deep part of the ligament is most commonly damaged out of the two. Injury to this ligament occurs when the force is applied from the lateral side of the knee when the leg is in a fixed position [53]. Ultrasonography shows this injury as heterogeneously hypoechoic mass lesions due to the presence of edema and hemorrhage and also, there is a loss of sharply defined margins of the ligaments [54]. There is thickening of the whole ligament. According to a study, the sensitivity of sonography for medial Collateral ligament injuries was found to be nearly $94 \%$.

\section{Lateral Collateral Ligaments}

The lateral collateral ligament originates from the lateral condyle of the femur to the fibular head. Lateral collateral ligament injury occurs when there is the application of force on the inside of the knee which causes the ligament to overstretch or tear. A partial tear shows tendon thickening while a complete tear will show hypoechoic focal hematoma and destruction of the fibers of the ligament.

\section{Cartilaginous}

In diseases affecting the cartilage, it is very important to assess the extent of damage to the cartilaginous structure for prompt treatment. Cartilage on ultrasound appears hypoechoic with a smooth contour. Ultrasound has a number of advantages over Magnetic resonance imaging in detecting cartilaginous thinning and minute surface defects.

\section{Osteoarthritis}

One of the most common joint disorders is osteoarthritis. It has become one of the most prevalent degenerative cartilaginous diseases affecting lifestyles of a huge population [55]. The most important measure taken to detect osteoarthritis is the thickening of the cartilage which represents the onset and the progression of the disease. There are also other associated conditions like the formation of osteophytes, erosion of the bones, and synovial lesions. Osteoarthritis is detected on ultrasonography as the decreased quantity of tibiofemoral cartilage, thinning of the hyaline cartilage $[56,57]$. Sometimes, bony lumps can also be seen in the knee floating around. Bony erosion will be revealed as hypoechoic like defects along the sharp margins of articular cartilage [58].

\section{Rheumatoid Arthritis}

Rheumatoid arthritis is an autoimmune disorder in which multiple joints of the body are affected by its own defenses. It is characterized by progressive disability, systemic complications, persistent synovial inflammation, and the presence of antibodies against the rheumatoid factor [59]. The T cells are found in abundance in the synovium of the joint but the pathophysiology behind destruction of the joints mediated by T-cells is still unknown. According to some studies, smoking is one of the most common environmental risk factors found in patients with rheumatoid arthritis [60]. Studies also link some genetic factors to rheumatoid arthritis. The morphological changes brought about are easily detectable by the use of ultrasound. Ultrasound helps us to monitor the response of the disease to the administered drugs [61, 62]. Increased synovial thickening and presence of bony erosions are some of the common findings in which conventional therapy does not do much better. Power Doppler ultrasonography also enables us to look for increased vascularity in the synovium. Hyperplasia of the synovium of the joint is the major contributor to cartilage damage. Nowadays, the treatment of rheumatoid arthritis has improved significantly over the recent decade owing to early diagnosis of the disease. Disease modifying anti-rheumatic agents has proven to be effective in its treatment after which tissue necrosis factor inhibitors are usually the second line of drugs in the treatment regimen.

\section{CONCLUSION}

Doppler ultrasonography has revolutionized our ways of diagnosing most diseases and also helps us with the necessary precise treatment. The development of high-resolution ultrasonography has made it one of the most commonly used imaging techniques in the field of orthology. Tendinopathies, ligament injuries, disease-causing degeneration of articular cartilage, complete or partial rupture of a tendon, synovial lesions or, inflammation of bursae, all can be assessed with maximum accuracy using ultrasonography. To cure any disease completely, there must be a whole understanding of the location and severity of the disease so that prompt treatment measures can be performed as early as possible. Doppler ultrasonography helps us in climbing this step.

\section{REFERENCES}

1. Bunt, C. W., Jonas, C. E., \& Chang, J. G. (2018). Knee pain in adults and adolescents: the initial evaluation. American family physician, 98(9), 576-585.

2. Nguyen, U. S. D., Zhang, Y., Zhu, Y., Niu, J., Zhang, B., \& Felson, D. T. (2011). Increasing prevalence of knee pain and symptomatic knee osteoarthritis: survey and cohort data. Annals of internal medicine, 155(11), 725-732. 
3. Turkiewicz, A., Gerhardsson de Verdier, M., Engström, G., Nilsson, P. M., Mellström, C., Lohmander, L. S., \& Englund, M. (2015). Prevalence of knee pain and knee OA in southern Sweden and the proportion that seeks medical care. Rheumatology, 54(5), 827-835.

4. Inoue, R., Ishibashi, Y., Tsuda, E., Yamamoto, Y., Matsuzaka, M., Takahashi, I., ... \& Toh, S. (2011). Knee osteoarthritis, knee joint pain and aging in relation to increasing serum hyaluronan level in the Japanese population. Osteoarthritis and cartilage, 19(1), 51-57.

5. Magnusson, K., Kumm, J., Turkiewicz, A., \& Englund, M. (2018). A naturally aging knee, or development of early knee osteoarthritis?. Osteoarthritis and cartilage, 26(11), 1447-1452.

6. Felson, D. T., Naimark, A., Anderson, J., Kazis, L., Castelli, W., \& Meenan, R. F. (1987). The prevalence of knee osteoarthritis in the elderly. The Framingham Osteoarthritis Study. Arthritis \& Rheumatism: Official Journal of the American College of Rheumatology, 30(8), 914-918.

7. Ho-Pham, L. T., Lai, T. Q., Mai, L. D., Doan, M. C., Pham, H. N., \& Nguyen, T. V. (2014). Prevalence of radiographic osteoarthritis of the knee and its relationship to self-reported pain. PloS one, 9(4), e94563.

8. McNally, E. G. (2001). Imaging assessment of anterior knee pain and patellar maltracking. Skeletal Radiology, 30(9), 484-495.

9. Boegård, T. L., Rudling, O., Petersson, I. F., \& Jonsson, K. (2001). Magnetic resonance imaging of the knee in chronic knee pain. A 2-year followup. Osteoarthritis and cartilage, 9(5), 473-480.

10. Disler, D. G. (2011). Articular cartilage in the knee: current MR imaging techniques and applications in clinical practice and research. Invited commentary. Radiographics: a review publication of the Radiological Society of North America, Inc, 31(1), 61-62.

11. Chang, K. V., Huang, Y. C., \& Wu, W. T. (2021). Ultrasound imaging for a male with anterior knee pain. J Med Ultrasound.

12. Sowers, M. F., Hayes, C., Jamadar, D., Capul, D., Lachance, L., Jannausch, M., \& Welch, G. (2003). Magnetic resonance-detected subchondral bone marrow and cartilage defect characteristics associated with pain and X-ray-defined knee osteoarthritis. Osteoarthritis and Cartilage, 11(6), 387-393.

13. Sweetman, G. M., Crawford, G., Hird, K., \& Fear, M. W. (2013). The benefits and limitations of using ultrasonography to supplement anatomical understanding. Anatomical sciences education, 6(3), 141-148.

14. Mathew, A. J., Danda, D., \& Conaghan, P. G. (2016). MRI and ultrasound in rheumatoid arthritis. Current opinion in rheumatology, 28(3), 323-329.

15. Wang, S. Y., Olson-Kellogg, B., Shamliyan, T. A., Choi, J. Y., Ramakrishnan, R., \& Kane, R. L. (2012). Physical therapy interventions for knee pain secondary to osteoarthritis: a systematic review. Annals of internal medicine, 157(9), 632644.

16. Roberts, C. S., Beck Jr, D. J., Heinsen, J., \& Seligson, D. (2002). Review article diagnostic ultrasonography: applications in orthopaedic surgery. Clinical Orthopaedics and Related Research®, 401, 248-264.

17. Waldman, S. D. (2019). Superficial Infrapatellar Bursitis. In: Atlas of Common Pain Syndromes. Elsevier; 453-455.

18. Waldman, S. D. (2019). Prepatellar Bursitis. In: Atlas of Common Pain Syndromes. Elsevier; p. 449-452

19. Baumbach, S. F., Lobo, C. M., Badyine, I., Mutschler, W., \& Kanz, K. G. (2014). Prepatellar and olecranon bursitis: literature review and development of a treatment algorithm. Archives of orthopaedic and trauma surgery, 134(3), 359-370.

20. Mysnyk, M. C., Wroble, R. R., Foster, D. T., \& Albright, J. P. (1986). Prepatellar bursitis in wrestlers. The American journal of sports medicine, 14(1), 46-54.

21. Artul, S., Khazin, F., Hakim, J., \& Habib, G. (2014). Ultrasonographic findings in a large series of patients with knee pain. Journal of clinical imaging science, 4(45):45.

22. Khodaee, M. (2017). Common superficial bursitis. American family physician, 95(4), $224-$ 231.

23. Waldman, S. D. (2019). Pes Anserine Bursitis. In: Atlas of Common Pain Syndromes. Elsevier; p. 468-471.

24. Rennie, W. J., \& Saifuddin, A. (2005). Pes anserine bursitis: incidence in symptomatic knees and clinical presentation. Skeletal radiology, 34(7), 395-398.

25. Uysal, F., Akbal, A., Gökmen, F., Adam, G., \& Reşorlu, M. (2015). Prevalence of pes anserine bursitis in symptomatic osteoarthritis patients: an ultrasonographic prospective study. Clinical rheumatology, 34(3), 529-533.

26. Imani, F., Rahimzadeh, P., Gharehdag, F. A., \& Faiz, S. H. R. (2013). Sonoanatomic variation of pes anserine bursa. The Korean journal of pain, 26(3), 249-254.

27. Powers, J. M., \& Ray, T. (2021). Popliteal Cyst. In: Common Pediatric Knee Injuries. Cham: Springer International Publishing; p. 191-196.

28. Kim, J. S., Lim, S. H., Hong, B. Y., \& Park, S. Y. (2014). Ruptured popliteal cyst diagnosed by ultrasound before evaluation for deep vein thrombosis. Annals of rehabilitation medicine, 38(6), 843-846. 
29. Labropoulos, N., Shifrin, D. A., \& Paxinos, O. (2004). New insights into the development of popliteal cysts. British journal of surgery, 91(10), 1313-1318.

30. Pathria, M. N., Zlatkin, M., Sartoris, D. J., Scheible, W., \& Resnick, D. (1988). Ultrasonography of the popliteal fossa and lower extremities. Radiologic Clinics of North America, 26(1), 77-85.

31. Osgood-Schlatter's Disease. In: Pediatric Practice Guidelines. New York, NY: Springer Publishing Company; 2020.

32. Czyrny, Z. (2010). Osgood-Schlatter disease in ultrasound diagnostics-a pictorial essay. Medical ultrasonography, 12(4), 323-335.

33. Vreju, F., Ciurea, P., \& Rosu, A. (2010). OsgoodSchlatter disease-ultrasonographic diagnostic. Medical ultrasonography, 12(4), 336339 .

34. Blankstein, A., Cohen, I., Heim, M., Salai, M., Chechick, A., Ganel, A., \& Diamant, L. (2001). Ultrasonography as a diagnostic modality in Osgood-Schlatter disease. Archives of orthopaedic and trauma surgery, 121(9), 536-539.

35. Blankstein, A., Cohen, I., Heim, M., Salai, M., Chechick, A., Ganel, A., \& Diamant, L. (2001). Ultrasonography as a diagnostic modality in Osgood-Schlatter disease. Archives of orthopaedic and trauma surgery, 121(9), 536-539.

36. Waldman, S. D. (2019). Jumper's Knee. In: Atlas of Common Pain Syndromes. Elsevier; p. 435439.

37. Lian, Ø. B., Engebretsen, L., \& Bahr, R. (2005). Prevalence of jumper's knee among elite athletes from different sports: a cross-sectional study. The American journal of sports medicine, 33(4), 561567.

38. Myllymäki, T., Bondestam, S., Suramo, I., Cederberg, A., \& Peltokallio, P. (1990). Ultrasonography of jumper's knee. Acta Radiologica, 31(2), 147-149.

39. Khan, K. M., Cook, J. L., Kiss, Z. S., Visentini, P. J., Fehrmann, M. W., Harcourt, P. R., ... \& Wark, J. D. (1997). Patellar tendon ultrasonography and jumper's knee in female basketball players: a longitudinal study. Clinical journal of sport medicine: official journal of the Canadian Academy of Sport Medicine, 7(3), 199-206.

40. Fritschy, D., \& de Gautard, R. (1993). Jumper's Knee and Ultrasonography. J Orthop Med. 15(3):70-3.

41. Fritschy, D. (1988). Jumper's Knee Ultrasonography. In: Surgery and Arthroscopy of the Knee. Berlin, Heidelberg: Springer Berlin Heidelberg; 495-497.

42. Razek, A. A. K. A., Fouda, N. S., Elmetwaley, N., \& Elbogdady, E. (2009). Sonography of the knee joint. Journal of ultrasound, 12(2), 53-60.
43. Mullis, B. H., \& Gaski, G. E., editors. (2020). 39 Quadriceps and Patellar Tendon Ruptures. In: Synopsis of Orthopaedic Trauma Management. Stuttgart: Georg Thieme Verlag.

44. Reilly, J. (2010). Quadriceps and Patellar Tendon Ruptures. In: The Knee. World Scientific; 313 322.

45. LaRocco, B. G., Zlupko, G., \& Sierzenski, P. (2008). Ultrasound diagnosis of quadriceps tendon rupture. The Journal of emergency medicine, 35(3), 293-295.

46. Prado-Costa, R., Rebelo, J., Monteiro-Barroso, J., \& Preto, A. S. (2018). Ultrasound elastography: compression elastography and shear-wave elastography in the assessment of tendon injury. Insights into Imaging, 9(5), 791-814.

47. Jacobson, J. A., Ruangchaijatuporn, T., Khoury, V., \& Magerkurth, O. (2017, April). Ultrasound of the knee: common pathology excluding extensor mechanism. In Seminars in musculoskeletal radiology (Vol. 21, No. 02, pp. 102-112). Thieme Medical Publishers.

48. Wang, J., Wu, H., Dong, F., Li, B., Wei, Z., Peng, Q., .. \& Xu, J. (2018). The role of ultrasonography in the diagnosis of anterior cruciate ligament injury: a systematic review and meta-analysis. European journal of sport science, 18(4), 579-586.

49. Kumar, S., Kumar, A., Kumar, S., \& Kumar, P. (2018). Functional ultrasonography in diagnosing anterior cruciate ligament injury as compared to magnetic resonance imaging. Indian journal of orthopaedics, 52, 638-644.

50. Grzelak, P., Podgórski, M. T., Stefańczyk, L., \& Domżalski, M. (2015). Ultrasonographic test for complete anterior cruciate ligament injury. Indian journal of orthopaedics, 49, 143-149.

51. Schüttler, K. F., Ziring, E., Ruchholtz, S., \& Efe, T. (2017). Verletzungen des hinteren Kreuzbands. Der Unfallchirurg, 120(1), 55-68.

52. Wilson, M. P., Nobbee, D., Lui, K., \& Low, G. (2021). The diagnostic accuracy of knee ultrasound for anterior cruciate ligament and posterior cruciate ligament injuries is likely overestimated. SN Compr Clin Med. 3(1):75-76.

53. Lundblad, M., Hägglund, M., Thomeé, C., Senorski, E. H., Ekstrand, J., Karlsson, J., \& Waldén, M. (2019). Medial collateral ligament injuries of the knee in male professional football players: a prospective three-season study of 130 cases from the UEFA Elite Club Injury Study. Knee Surgery, Sports Traumatology, Arthroscopy, 27(11), 3692-3698.

54. Tanaka, K., Okamoto, Y., Makihara, T., Maehara, K., Yoshizawa, T., Minami, M., \& Yamazaki, M. (2017). Clinical interpretation of asymptomatic medial collateral ligament injury observed on magnetic resonance imaging in adolescent 
baseball players. Japanese journal of radiology, 35(6), 319-326.

55. Vina, E. R., \& Kwoh, C. K. (2018). Epidemiology of osteoarthritis: literature update. Current opinion in rheumatology, 30(2), 160.

56. Daniel, M. S., Oo, W. M., Wang, X., Deveza, L. A., Linklater, J., \& Hunter, D. J. (2019). Validity of musculoskeletal ultrasonography in measuring joint effusion in knee osteoarthritis. Osteoarthritis and Cartilage, 27, S370-S371.

57. Nevalainen, M. T., Pylväläinen, J., Kauppinen, K., Pamilo, K., Pesola, M., \& Koski, J. (2018). Ultrasonography of the late-stage knee osteoarthritis prior total knee arthroplasty: comparison of the ultrasonographic, radiographic and intra-operative findings. Osteoarthritis Cartilage. 26:S445-6.

58. Chao, J., \& Kalunian, K. (2008). Ultrasonography in osteoarthritis: recent advances and prospects for the future. Current opinion in rheumatology, 20(5), 560-564.
59. Sayah, A., \& English III, J. C. (2005). Rheumatoid arthritis: a review of the cutaneous manifestations. Journal of the American Academy of Dermatology, 53(2), 191-209.

60. Chang, K., Yang, S. M., Kim, S. H., Han, K. H., Park, S. J., \& Shin, J. I. (2014). Smoking and rheumatoid arthritis. International journal of molecular sciences, 15(12), 22279-22295.

61. Ghib, L. J., Tamas, M. M., Damian, L. O., Felea, I., Muntean, L. M., Rednic, N., \& Rednic, S. (2015). The role of ultrasonography in assessing disease activity in patients with rheumatoid arthritis and associated fibromyalgia. Medical ultrasonography, 17(3), 339-344.

62. Qvistgaard, E., Røgind, H., Torp-Pedersen, S., Terslev, L., Danneskiold-Samsøe, B., \& Bliddal, H. (2001). Quantitative ultrasonography in rheumatoid arthritis: evaluation of inflammation by Doppler technique. Annals of the rheumatic diseases, 60(7), 690-693. 